\title{
SYMMETRIC MEANS AND MATRIX INEQUALITIES
}

\section{PETER BULLEN AND MARVIN MARCUS ${ }^{1}$}

1. Introduction. The purpose of this note is to present several new inequalities for elementary symmetric functions and exploit these to investigate the inequalities that exist among the eigenvalues of submatrices and sums of positive definite hermitian (p.d.h.) matrices.

We describe our notation: $(a)=\left(a_{1}, \cdots, a_{n}\right)$ will denote an $n$-tuple of positive numbers, $\left(a_{i}^{\prime}\right)$ is the $(n-1)$-tuple obtained by deleting $a_{i}$ from $(a) . E_{r}(a)$ denotes the $r$ th elementary symmetric function of the $(a)$ :

$$
E_{r}(a)=\sum_{1 \leq i_{1}<\cdots<i_{r} \leq n} \prod_{j=1}^{r} a_{i_{j}}, \quad E_{0}=1 .
$$

$p_{r}(a)$ is the mean of $E_{r}(a)$,

$$
p_{r}=p_{r}(a)=\left(\begin{array}{l}
n \\
r
\end{array}\right)^{-1} E_{r}(a) .
$$

If $(a)=\left(a_{1}, \cdots, a_{n}\right)$ then $(\bar{a})=\left(a_{1}, \cdots, a_{n}, a_{n+1}\right)$ and $p_{r}\left(a_{1}, \cdots, a_{n+1}\right)$ $=p_{r}$. If two sets $(a)$ and $(b)$ are related by $a_{i}=\lambda b_{i}, \lambda>0, i=1, \cdots, n$ then we say ( $a$ ) is proportional to (b). We note that if $A$ is an $n$-square complex matrix with eigenvalues $\alpha_{1}, \cdots, \alpha_{n}$ then $(-1)^{r} E_{r}(\alpha)$ is the coefficient of $x^{n-r}$ in the characteristic polynomial $\operatorname{det}(x I-A)$.

2. Results.

ThEOREM 1. If $1 \leqq r \leqq k \leqq n$ then

$$
p_{r}^{k} / p_{k}^{r} \leqq p_{r}^{k+1} / p_{k+1}^{r},
$$

with equality if and only if $a_{1}=\cdots=a_{n+1}$.

We give two proofs.

Proof 1. The following results are known $[2$, p. 52]

$$
p_{s}=\frac{n+1-s}{n+1} p_{s}+\frac{s}{n+1} a_{n+1} p_{s-1},
$$

(3) if $s<t$ then $p_{s}^{1 / s} \geqq p_{t}^{1 / t}$ with equality if and only if $a_{1}=\cdots=a_{n}$,

(4) if $a_{1}=\cdots=a_{n}=\alpha$ then

Presented to the Society, January 24, 1961; received by the editors May 24, 1960.

1 The work of this author was supported by U. S. Air Force Contract No. AF $49(638)-776$. 


$$
p_{s}=\frac{n+1-s}{n+1} \alpha^{s}+\frac{s}{n+1} a_{n+1} \alpha^{s-1} .
$$

If $r=k$ then (1) is implied by (3) so we assume $r<k$. Using (2) with both $s=p$ and $s=k+1$ (1) is seen to be equivalent to

$$
\begin{aligned}
L & =\left\{\frac{n-k}{n+1}\left(p_{k+1} / p_{k}\right)+a_{n+1} \frac{k+1}{n+1}\right\}^{r} p_{r}^{k} \\
& \leqq\left\{\frac{n+1-r}{n+1} p_{r}+\frac{r}{n+1} a_{n+1} p_{r-1}\right\}^{k+1}=R .
\end{aligned}
$$

By (3)

$$
\begin{aligned}
& L \leqq\left\{\frac{n-k}{n+1} p_{k}^{1 / k}+a_{n+1} \frac{k+1}{n+1}\right\}^{r} p_{r}^{k}, \\
& R \geqq\left\{\frac{n+1-r}{n+1} p_{r}+\frac{r}{n+1} a_{n+1} p_{r}^{(r-1) / r}\right\}^{k+1} .
\end{aligned}
$$

By (4) with $\alpha=p_{r}^{1 / r}$ the right-hand side of (5) is the mean of the $r$ th elementary symmetric function of the numbers $a_{1}=a_{2}=\cdots=a_{n}$ $=p_{r}^{1 / r}$ and $a_{n+1}$. Hence by (3) with $t=r$ and $s=k+1 R$ is not less than

$$
\begin{aligned}
\left\{\frac{n-k}{n+1} p_{r}^{(k+1) / r}+\frac{k+1}{n+1} p_{r}^{k / r} a_{n+1}\right\}^{r} & =p_{r}^{k}\left\{\frac{n-k}{n+1} p_{r}^{1 / r}+\frac{k+1}{n+1} a_{n+1}\right\}^{r} \\
& \geqq L .
\end{aligned}
$$

This proves the inequality. The inequality is strict in (3) and hence in our arguments unless $a_{1}=\cdots=a_{n}=p_{r}^{1 / r}=a_{n+1}$, completing the proof.

Proof. 2. In this argument we show that the right-hand side of (1) is a convex function of $a_{n+1}$ with at most one critical value in $a_{n+1}>0$.

Let $x=a_{n+1}$ and set

$$
\phi(x)=\bar{p}_{r}^{k+1} / \bar{p}_{k+1}^{r} \text {. }
$$

Since $\phi(x)$ is of the form $(x+a)^{k+1} /(x+b)^{r}$ to within a positive constant multiple and $k>r(k=r$ is again an application of (3)) we can check that $\phi^{\prime \prime}(x)>0$ for $x \geqq 0$ and hence $\phi(x)$ is convex. Thus it suffices to show that

$$
\phi(0) \geqq p_{r}^{k} / p_{k}^{r},
$$

and 


$$
\phi\left(x_{0}\right) \geqq p_{r}^{k} / p_{k}^{r} \quad \text { when } \quad \phi^{\prime}\left(x_{0}\right)=0, \quad x_{0}>0 .
$$

Now

$$
\phi(0)=\frac{(n+1-r)^{k+1}}{(n+1)^{k+1-r}(n-k)^{r}} p_{r}^{k+1} / p_{k+1}^{r}
$$

and by (3) we need only check that

$$
(n+1-r)^{k+1}>(n-k)^{r}(n+1)^{k+1-r}
$$

whenever $1 \leqq r \leqq k \leqq n$. If $k=n$ (8) is clear, otherwise let $K=k+1$, $N=n+1$ and (8) becomes

$$
\left(1-\frac{r}{N}\right)^{K}>\left(1-\frac{K}{N}\right)^{r}, \quad \frac{r}{N}<\frac{K}{N}<1 .
$$

Taking natural logarithms of both sides and expanding, (9) is immediately implied by $r<K$. We next see that $\phi^{\prime}\left(x_{0}\right)=0$ and $x_{0} \geqq 0$ implies

$$
x_{0}=\frac{(n+1-r) p_{r} p_{k}-(n-k) p_{k+1} p_{r-1}}{(k+1-r) p_{k} p_{r-1}}
$$

and hence

$$
\begin{aligned}
\phi\left(x_{0}\right)= & \left(p_{r}^{k} / p_{k}^{r}\right)\left(p_{r-1}^{r} / p_{r}^{r-1}\right) \\
& \cdot\left[\frac{(k+1)(n+1-r)-r(n-k)\left(p_{r-1} / p_{r}\right)\left(p_{k+1} / p_{k}\right)}{(n+1)(k+1-r)}\right]^{k+1-r} .
\end{aligned}
$$

By (3) it suffices to show that the quantity in the square brackets is greater than 1. This is equivalent to

$$
p_{r-1} p_{k+1} \leqq p_{r} p_{k}
$$

which is established by an easy induction on $k-r$ from the case $k=r[2$, p. 52]. Now, the only time that equality can hold in (3) and (10) is for $a_{1}=\cdots=a_{n}=a$. But then we check that $x_{0}=a$ also. The proof is complete.

CoROllary. If $\mathfrak{A}_{n}$ and $G_{n}$ are the arithmetic and geometric means of (a) then

$$
\left(\mathfrak{A}_{n} / G_{n}\right)^{n} \leqq\left(\mathfrak{A}_{n+1} / G_{n+1}\right)^{n+1}
$$

with equality if and only if $a_{1}=\cdots=a_{n+1}$.

This result is an analogue of an inequality due to $R$. Rado [2, p. 61]: 


$$
n\left(\mathfrak{A}_{n}-G_{n}\right) \leqq(n+1)\left(\mathfrak{A}_{n+1}-G_{n+1}\right) .
$$

The inequality (12) can be derived in the same way as (11) was from (3) with $s=n$ and $t=1$.

TheOREM 2. Let $H$ be a p.d.h. $(n+1)$-square matrix and let $K$ be an $n$-square principal submatrix of $H$. If $\mu_{1} \geqq \cdots \geqq \mu_{n+1}>0$ and $\lambda_{1} \geqq \cdots$ $\geqq \lambda_{n}>0$ are the eigenvalues of $H$ and $K$ respectively then

$$
\begin{aligned}
&\left(p_{1}^{k}\left(\lambda_{1}, \cdots, \lambda_{n}\right) / p_{k}\left(\lambda_{1}, \cdots, \lambda_{n}\right)\right) \\
& \leqq\left(p_{1}^{k+1}\left(\mu_{1}, \cdots, \mu_{n+1}\right) / p_{k+1}\left(\mu_{1}, \cdots, \mu_{n+1}\right)\right) .
\end{aligned}
$$

Proof. Let $K$ be obtained from $H$ by deleting row and column $i$ of $H$. Let $x_{1}, \cdots, x_{n}$ be an orthonormal (o.n.) set of $n$-dimensional eigenvectors of $K$. Let $y_{j}, j=1, \cdots, n$, be the set of $(n+1)$-vectors given by

$$
y_{j}=\left(x_{j 1}, \cdots, x_{j i-1}, 0, x_{j i}, \cdots, x_{j n}\right),
$$

where $x_{j}=\left(x_{j 1}, \cdots, x_{j i-1}, x_{j i}, \cdots, x_{j n}\right)$ and let $y_{n+1}$ be the $(n+1)$ vector with 1 in position $i$, zero elsewhere. Then we check easily that $y_{1}, \cdots, y_{n+1}$ is an o.n. set of $(n+1)$-vectors and moreover

$$
\left(B y_{j}, y_{j}\right)=\left(K x_{j}, x_{j}\right), \quad j=1, \cdots, n \text {. }
$$

Then

$$
\begin{gathered}
\left(p_{1}^{k}\left(\lambda_{1}, \cdots, \lambda_{n}\right) / p_{k}\left(\lambda_{1}, \cdots, \lambda_{n}\right)\right)=\frac{\left(\sum_{j=1}^{n}\left(K x_{j}, x_{j}\right) / n\right)^{k}}{p_{k}\left(\left(K x_{1}, x_{1}\right), \cdots,\left(K x_{n}, x_{n}\right)\right)} \\
\leqq \frac{\left(\sum_{j=1}^{n+1}\left(H y_{j}, y_{j}\right) /(n+1)\right)^{k+1}}{p_{k+1}\left(\left(B y_{1}, y_{1}\right), \cdots,\left(B y_{n+1}, y_{n+1}\right)\right)} \\
\leqq\left(p_{1}^{k+1}\left(\mu_{1}, \cdots, \mu_{n+1}\right) / p_{k+1}\left(\mu_{1}, \cdots, \mu_{n+1}\right)\right) .
\end{gathered}
$$

The inequalities above follow from:

(i) the sum of the quadratic forms over a complete o.n. set is the trace; (ii) $p_{k+1}\left(\left(H y_{1}, y_{1}\right), \cdots,\left(H y_{n+1}, y_{n+1}\right)\right) \geqq p_{k+1}\left(\mu_{1}, \cdots, \mu_{n+1}\right)$ for any o.n. set $y_{1}, \cdots, y_{n+1}[4]$.

COROLlaRY. If $\tau_{k}$ and $d_{k}$ be the trace and determinant of $H_{k}$, the $k$ square principal submatrix of $H$ whose elements lie in the first $k$ rows and columns of $H$, then 


$$
\frac{\left(\tau_{k} / k\right)^{k}}{d_{k}} \leqq \frac{\left(\tau_{k+1} /(k+1)\right)^{k+1}}{d_{k+1}} .
$$

It might be conjectured that Theorem 2 follows from the Cauchy inequalities $[1$, p. 75$]$. However a proof via this route is made difficult by the fact that $p_{r}^{k} / p_{k}^{r}$ is not an increasing function.

In [5] it was proved that $E_{r} / E_{r-1}$ and $E_{r}^{t / r}$ are both concave functions for positive variables. We extend these results as follows.

THEOREM 3. If $1 \leqq p \leqq r \leqq n$ and $F_{r, p}=\left(E_{r} / E_{r-p}\right)^{1 / p}$ then

$$
F_{r, p}(a+b) \geqq F_{r, p}(a)+F_{r, p}(b)
$$

with equality if and only if $r=1, p=1$ or (a) is proportional to (b). Moreover, $F_{r, p}$ is a nondecreasing function of each $a_{j}$.

Proof.

$$
\begin{aligned}
F_{r, p}(a+b) & =\left(\prod_{j=1}^{p} E_{r-j+1}(a+b) / E_{r-j}(a+b)\right)^{1 / p} \\
& \geqq\left\{\prod_{j=1}^{p}\left(E_{r-j+1}(a) / E_{r-j}(a)\right)+\left(E_{r-j+1}(b) / E_{r-j}(b)\right)\right\}^{1 / p} \\
& \geqq\left\{\prod_{j=1}^{p} E_{r-j+1}(a) / E_{r-j}(a)\right\}^{1 / p}+\left\{\prod_{j=1}^{p} E_{r-j+1}(b) / E_{r-j}(b)\right\}^{1 / p} \\
& =F_{r, p}(a)+F_{r, p}(b) .
\end{aligned}
$$

The first inequality above is the result in [5] and inequality can hold if and only if $(a)$ is proportional to $(b)$. The second inequality is the Hölder inequality. Conversely if $(a)$ is proportional to $(b)$ the equality is easily checked.

We next compute

$$
\frac{\partial}{\partial a_{1}}\left\{E_{r}(a) / E_{r-p}(a)\right\}=\left\{E_{r-p}(a) E_{r-1}\left(a_{1}^{\prime}\right)-E_{r}(a) E_{r-p-1}\left(a^{\prime}\right)\right\} / E_{r-p}^{2}(a) .
$$

Now by (2) the numerator in this last expression becomes

$$
E_{r-1}\left(a_{1}^{\prime}\right) E_{r-p}\left(a_{1}^{\prime}\right)-E_{r}\left(a_{1}^{\prime}\right) E_{r-p-1}\left(a_{1}^{\prime}\right) \geqq 0 .
$$

This last inequality is found in [2, p. 52].

We apply Theorem 3 to obtain

THEOREM 4. If $A$ and $B$ are $n$-square p.d.h. matrices with eigenvalues $0<\alpha_{1} \leqq \cdots \leqq \alpha_{n}, 0<\beta_{1} \leqq \cdots \leqq \beta_{n}$ respectively and $C=A+B$ has eigenvalues $0<\delta_{1} \leqq \cdots \leqq \delta_{n}$, then for $1 \leqq p \leqq r \leqq k \leqq n$, 


$$
F_{r, p}\left(\delta_{1}, \cdots, \delta_{k}\right) \geqq F_{r, p}\left(\alpha_{1}, \cdots, \alpha_{k}\right)+F_{r, p}\left(\beta_{1}, \cdots, \beta_{k}\right) .
$$

Proof. By Theorem 3 and the fact that $F_{r, p}$ is homogeneous of degree 1 we conclude that $F_{r, p}$ is concave for positive variables. By a general extremal result in [3] it follows that if $x_{1}, \cdots, x_{k}$ are an o.n. set of vectors then

$$
F_{r, p}\left(\left(A x_{1}, x_{1}\right), \cdots,\left(A x_{p}, x_{p}\right)\right) \geqq F_{r, p}\left(\alpha_{i_{1}}, \cdots, \alpha_{i_{k}}\right)
$$

for some index set $1 \leqq i_{1}<\cdots<i_{k} \leqq n$. But since $F_{r, p}$ is nondecreasing we know that

$$
F_{r, p}\left(\alpha_{i_{1}}, \cdots, \alpha_{i_{k}}\right) \geqq F_{r, p}\left(\alpha_{1}, \cdots, \alpha_{k}\right) .
$$

Now select $x_{1}, \cdots, x_{k}$ to be an o.n. set of eigenvectors of $C$ corresponding to $\delta_{1}, \cdots, \delta_{k}$ respectively. Then

$$
\begin{aligned}
& F_{r, p}\left(\delta_{1}, \cdots, \delta_{k}\right)=F_{r, p}\left(\left(C x_{1}, x_{1}\right), \cdots,\left(C x_{k}, x_{k}\right)\right) \\
& \quad=F_{r, p}\left(\left(A x_{1}, x_{1}\right)+\left(B x_{1}, x_{1}\right), \cdots,\left(A x_{k}, x_{k}\right)+\left(B x_{k}, x_{k}\right)\right) \\
& \quad \geqq F_{r, p}\left(\left(A x_{1}, x_{1}\right), \cdots,\left(A x_{k}, x_{k}\right)\right)+F_{r, p}\left(\left(B x_{1}, x_{1}\right), \cdots,\left(B x_{k}, x_{k}\right)\right) \\
& \quad \geqq F_{r, p}\left(\alpha_{1}, \cdots, \alpha_{k}\right)+F_{r, p}\left(\beta_{1}, \cdots, \beta_{k}\right)
\end{aligned}
$$

in which we have used Theorem 3 and (16) and (17) in succession.

CorollaRy. If $x^{n}+\sum_{j=1}^{n}(-1)^{i} c_{j}(A) x^{n-j}$ is the characteristic polynomial of $A$ then

$$
\left(c_{r}(C) / c_{r-p}(C)\right)^{1 / p} \geqq\left(c_{r}(A) / c_{r-p}(A)\right)^{1 / p}+\left(c_{r}(B) / c_{r-p}(B)\right)^{1 / p} .
$$

PRoof. Take $k=n$ in Theorem 4.

\section{REFERENCES}

1. H. L. Hamburger and M. E. Grimshaw, Linear transformations in n-dimensional vector space, Cambridge, University Press, 1951.

2. G. H. Hardy, J. E. Littlewood, and G. Polya, Inequalities (2nd ed.), Cambridge University Press, 1952.

3. M. Marcus, Convex functions of quadratic forms, Duke Math. J. vol. 24 (1957) pp. 321-326.

4. M. Marcus and J. L. McGregor, Extremal properties of Hermitian matrices, Canad. J. Math. vol. 8 (1956) pp. 524-531.

5. M. Marcus and L. Lopes, Inequalities for symmetric functions and Hermitian matrices, Canad. J. Math. vol. 9 (1957) pp. 305-312.

The University of British Columbia, Vancouver, Canada 